\title{
Metal Nanostructure Synthesis via Surface Energy
}

\section{Driven Growth.}

Curtis J. O'Kelly, Soon Jung Jung, and John J. Boland*

School of Chemistry, Trinity College Dublin, Dublin 2, Ireland

CRANN Institute, Trinity College Dublin, Dublin 2, Ireland

Metal nanowire, metal synthesis, single crystal, SEDG, agglomeration, crystallization,

ABSTRACT: Identifying new synthesis methods to produce single crystal metal nanowires has the potential to expand nanowire based technology integration and increase applications. The catalogue of single crystal metal nanostructures is rather limited compared to the range of high aspect semiconductor nanowires synthesized via well-established vapor liquid solid and atomic layer deposition methods. The surface energy driven growth (SEDG) method opens new possibilities for producing novel metal nanostructures. The method, based on the relative surface energy of the components involved in the synthesis is presented as a standalone method for producing high aspect ratio single crystal metal nanowires. Wire growth is realized following eutectic temperature annealing of individual thin films that make up binary alloy systems. In response to thermal annealing the high surface energy component is observed to crystallize and form wire structures directly form the bilayer material without the introduction of growth precursors. The potential of this growth method has been demonstrated in the several recent examples of nanostructures synthesized using SEDG. Focus here will be on generalizing the method to enable future synthesis via this new method. Controlled positioning of the growth 
can be achieved through manipulation of dewetting phenomena to control mass flow and facilitated by the placement of defects that nucleate the de-wetting phenomenon. The physical principles dictating the growth method are covered in detail to enable fine control over positioning and dewetting during growth. The principles of the method are presented together in detail for the first time with the aim of increasing the accessibility of the method for the wider scientific community.

\section{Introduction}

Metallic nanowires with high aspect ratio and well defined crystal structure have great potential to increase performance in areas such as catalysis and nanowire networks for transparent conductors. Conventional metallic nanowires can vary in crystal quality depending on the nature of synthesis method and which ultimately impacts their physical properties and usefulness. Although high quality nanowires can be synthesized using "bottom-up" or "topdown" methods, precise control, placement and manipulation of theses nanowire remains achallenge. ${ }^{1}$ The surface energy driven growth method (SEDG) described herein presents opportunities for producing new single crystal metal wire materials and controlling the position of their growth on the substrate via liquid capillary dewetting effects.

The SEDG method is stand alone synthesis that shares some physical phenomena in common with VLS synthesis such as nucleation and growth from saturated droplets. In contrast to VLS however the surface energy of the components of the synthesis have a much greater role in defining and ultimately determining growth. VLS, introduced by Wagner ${ }^{2}$, involves the nucleation of precursor gases on a substrate with typically Au metal droplets acting as catalysts. The wealth of materials produced via this method is extensive ranging from semiconductors to oxides and insulators. Over the last 50 years tremendous advances have been made in 
expanding the breath of anisotropic materials that can be produced including elemental semiconductors ${ }^{3,4}$, II-VI semiconductors ${ }^{5,6}$, III-V semiconductors ${ }^{7,8}$, oxides ${ }^{9,10}$, nitrides ${ }^{11}$ and carbides ${ }^{12,13}$. The method is limited by the solubility of the precursor gas within the metal catalyst and has not yet been reported to produce metallic nanowires.

Thin film liquid dewetting dynamics is an established field that has seen renewed interest as the behaviour of ultra thin film films of materials on supporting substrates have become of increasing importance in emergent technologies. One notable example is single crystal siliconon-bulk oxide substrates, which is a critical material technology for next generation CMOS. The relative stability of films tens of nanometers thick are significantly reduced compared to films above $100 \mathrm{~nm}$ in thickness. This instability is evident by thermal processing which produces liquid dewetting patterns at temperatures far below the melting temperature of the bulk material. In the case of the SEDG method this thermal instability is leveraged to produce liquid-like instabilities in bilayer thin films far below their melting temperature. 


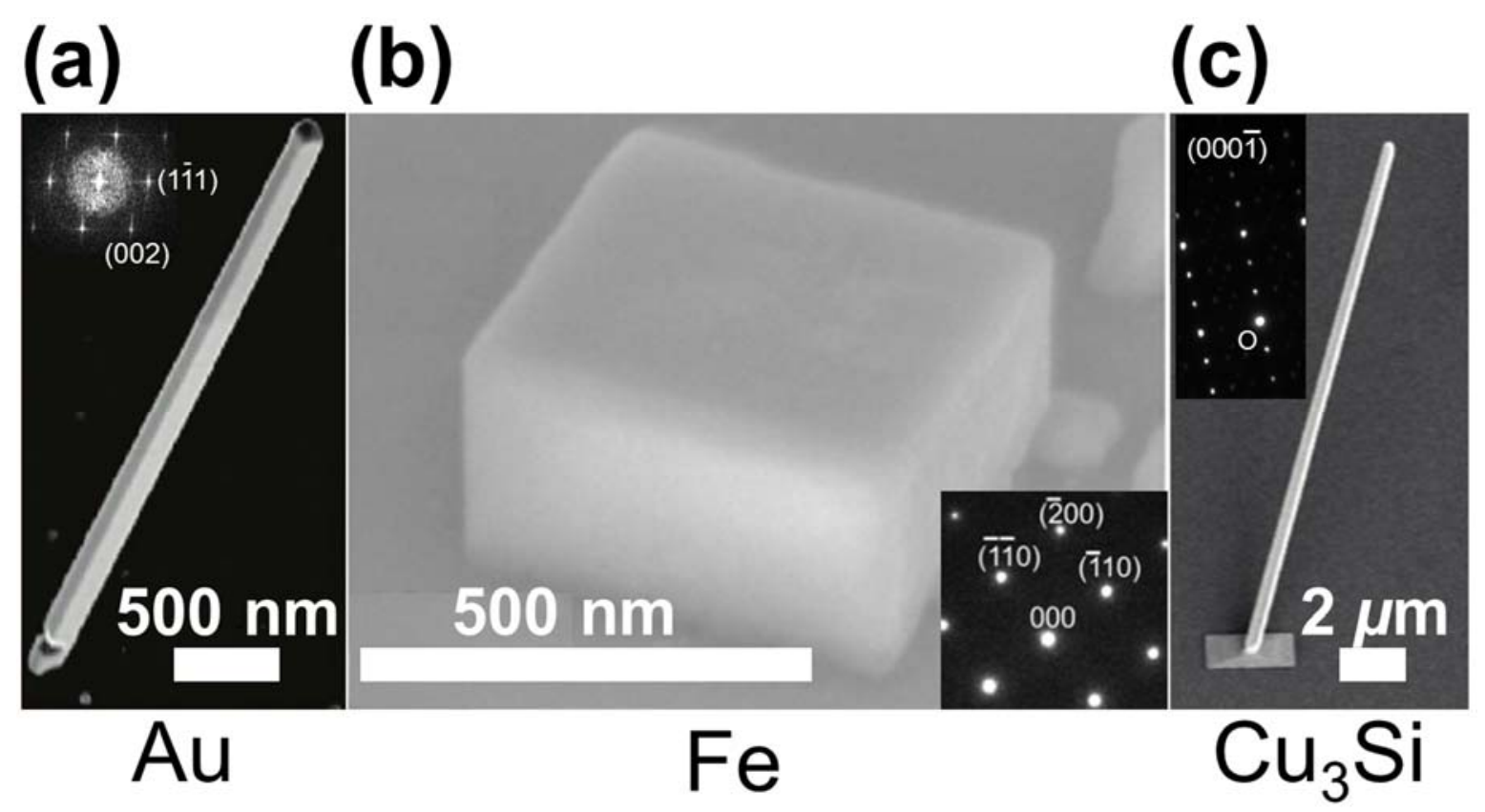

Figure 1 Three nanostructures grown via the SEDG method. (a) Au single crystal ${ }^{14}$. (b) Single crystal Fe nanocubes ${ }^{15}$. (c) Cu3Si nanowires ${ }^{16}$. Diffraction patterns for each are shown in the inset demonstrating the single crystal nature of the nanostructures grown via this method.

\section{Characteristics of the SEDG Method}

The method was first introduced by Jung et al for the Au-Ge system where it was discovered that heating thin films of $\mathrm{Au}$ and $\mathrm{Ge}$ at their eutectic temperature produced small heavily 
faceted particles and some extremely long Au nanowires $\sim 20$ um in length ${ }^{14}$. The two materials form a binary alloy system with a eutectic temperature of $\sim 350 \mathrm{C}$. The system is commonly investigated for the VLS synthesis of Ge nanowires ${ }^{17}$ using Ge precursor gas flux in the presence of a nanoscale Au film. It was therefore unexpected that Au nanowires should form upon heating of bilayer Au-Ge films. Heating the bilayer films resulted in a familiar pattern known as spinodal dewetting, resulting in the formation of agglomerated liquid phase particles. Many of the wires were observed to sprout from these agglomerated particles suggesting the particles behaved as nucleation and crystal growth centres for the newly formed wires, in a manner similar to that of VLS. It also was demonstrated that introducing ion beam lithography defined holes into the film prior to annealing made it possible to have a degree of control over the positioning and growth of the subsequently grown wires ${ }^{14}$. The phenomena led to the formation of 'agglomeration waves' but the physical origin was not understood at that time. A discussion of the physical origin of these 'agglomeration waves' is presented in this paper to develop new insight over the control of dewetting. This includes using our understanding of these waves to better predict film breakup, crystal positioning and growth. The merits of the method will be discussed in light of its accessibility and potential in fundamental studies of nucleation kinetics and morphology evolution performed in real time using a hot stage SEM platform. 


\section{Selection $\longrightarrow$ Deposition $\longrightarrow$ Process}

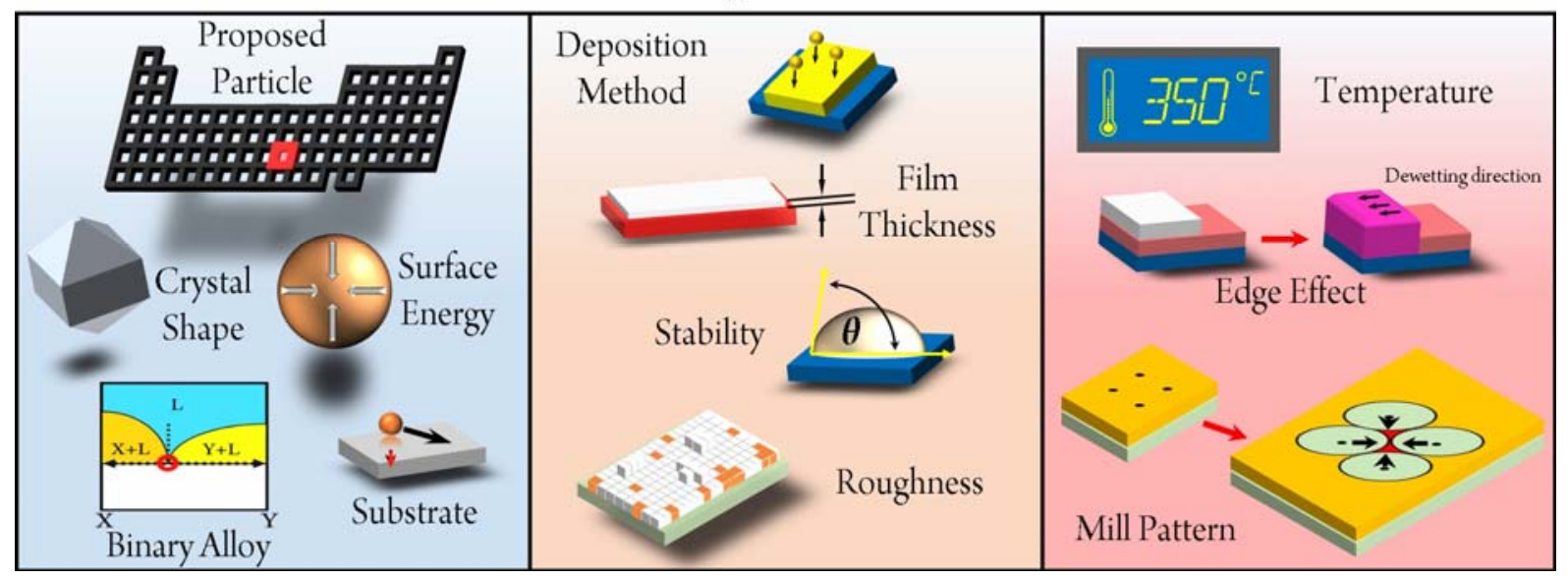

Figure 2 SEDG process flow chart depicting the various major design and process stages involved in growing nanostructures using this method.

Selection Stage involves choosing a candidate material to grow via SEDG. Transition metals should be easily produced by this method and although not pursued in this study it may be possible to synthesize some alloy nanomaterials also. Next the relevant pertinent physical data about the candidate material is collected the most important of which is the surface energy of the material as it has an impact on the liquid dynamics and nucleation later in the process. The shape of the candidate nanostructure produced with SEDG is determined by the lowest surface energy crystal facet of the material. Using Wulff construction will allow the final shape of the particle be determined at this point in the process. A complementary alloy must now be determined for the candidate material that has properties that facilitate growth. These include lower relative surface energy to the primary candidate material and a binary alloy phase diagram with a simple and accessible eutectic point. The eutectic temperature sets a processing temperature guideline for annealing. However, it is important to note the eutectic temperature in nanoscale materials can be significantly less than that of their bulk counterparts. Prior to stage 2 a substrate is chosen to accommodate growth. Typically the substrate should be inert with lower surface energy relative to the two binary alloy components which promotes dewetting of the liquidus alloy, a crucial step prior to nucleation. More complex SEDG approaches can include growth via substrate etching as demonstrate in the case of SEDG grown $\mathrm{Cu}_{3} \mathrm{Si}$ wires.

Deposition Stage Deposition of each alloy component can be performed by a variety of established methods including sputter deposition, electron beam evaporation and thermal evaporation. At this point film instabilities that promote growth can be introduced by using thin films and by the roughness of the deposited film. Introducing film edges during deposition also promotes local dewetting during processing.

Process Stage Sample processing is achieved by annealing the bilayer at temperatures close to the eutectic point in the binary alloy phase diagram. Prior to annealing, wire growth positioning can be realised by FIB milling a pattern of holes or defects into the bilayer to control dewetting and nucleation dynamics during film rupture and agglomeration. 


\section{Generalisation of SEDG Method}

To introduce the method we will apply the SEDG method to the synthesis of a generic material $\mathrm{X}$ with the goal of producing single crystal $\mathrm{X}$ nanostructures prepositioned on a substrate. Before experimental sample preparation begins the relevant physical properties of $\mathrm{X}$ must be collected and reviewed for SEDG suitability. The list of important parameters is described in the flow chart below - Figure 2. The most critical parameter in determining candidate materials is the surface energy of its most stable crystal plane (the crystal plane with the lowest surface energy value). This value will determine the ultimate shape of the crystal X as prescribed by Wulff construction of the nanostructure. For low index crystal planes such as $<100>,<110>$ and $<111>$ the final form of the crystal formed will be square, pyramidal and wire-like respectively, under equilibrium conditions. Novel single crystal nanostructures may be realized through selective material optimisation. More complicated crystal index planes could give rise to previously unreported nanostructure shapes such as nanowire shape of $\mathrm{Cu}_{3} \mathrm{Si}$ single crystal. ${ }^{16}$

The next step in the design flow is finding a suitable alloy material for $\mathrm{X}$. The surface energy of $\mathrm{X}$ must be greater than that of the alloying material $\mathrm{Y}$ for SEDG to be realised. Computational and experimental surface energy values of different materials along with their various crystal index planes are readily available in the literature ${ }^{18}$.

The nature of the $\mathrm{X}-\mathrm{Y}$ alloy is then scrutinized for the presence of readily accessible eutectic points in the binary alloy phase diagram. The eutectic point represents a special point in the XY binary alloy phase diagram where a set composition of the alloy melts and freezes at a single temperature. This temperature is typically well below that of the melting point of the individual bulk X and Y components. Moreover, the eutectic temperature of nanoscale films is typically well below that of the bulk materials. This is attractive from a process point of view because 
it allows the alloy to melt to form a liquid at 100 's C temperature instead of 1000 's C. ${ }^{19}$ Finding a suitable alloy candidate with a eutectic point in the binary phase diagram is perhaps the most constraining factor for materials synthesis via this method. The literature remains limited in the breath of binary alloy phase diagrams or each combination of material $\mathrm{X}$ with rest of the elements. This is a consequence of way binary phase diagrams are produced, through empirical data. The lack of binary alloy phase information may be offset by modelling and computer software which may help identify relevant eutectic alloy candidates for material X. It is worth mentioning that the nature of the binary phase diagram must be relatively simple. An overly complicated binary phase diagram with multiple eutectic points could potentially inhibit or frustrate the formation of the single crystal X nanostructure.

\section{Thin Film Stability}

The final design stage prior to sample preparation is to choose the substrate such that the liquidus eutectic alloy of $\mathrm{X}-\mathrm{Y}$ is morphologically unstable. This will promote film breakup followed by agglomeration. The morphological stability of a thin liquid film to breakup (dewetting) or to remain intact (wetting) on a given substrate is determined by the surface energy of the liquid film relative to the substrate. Youngs equation (1) is used to gauge the stability of liquids on a substrate either by directly measuring the equilibrium contact angle $\left(\theta_{\mathrm{CA}}\right)$ the liquid makes with the substrate or by estimating it using the surface energies of the three interfaces.

$$
\cos \theta_{C A}=\left(\frac{\gamma_{S V}-\gamma_{S L}}{\gamma_{L V}}\right)
$$


Where $\gamma_{\mathrm{SV}}, \gamma_{\mathrm{SL}}$ and $\gamma_{\mathrm{LV}}$ are the surface energies for the solid-vapour, solid-liquid and liquidvapour interfaces, Figure 3 (a) depicts a liquid droplet on a substrate. The equilibrium contact angle is used to estimate the tendency of a liquid to dewet a surface. By convention a large contact angle implies a greater tendency for the liquid to dewet the substrate. The value of contact angle may be interpreted as the liquids tendency to undergo a morphological transition from a film to an agglomerated/islanded state. Data on the surface energy of the eutectic liquidus alloy is extremely rare in almost all binary alloy liquids. It is further complicated by the fact the surface energy of an alloy liquid will change depending on the temperature and concentration of each component and which will change dynamically during annealing. ${ }^{20}$ This makes it difficult to predict the contact angle and therefore the stability of the liquidus eutectic alloy on a substrate. In the absence of this data using a very low surface energy substrate such as $\mathrm{Si}_{3} \mathrm{~N}_{4}, \mathrm{SiO}_{2}$ and $\mathrm{Al}_{2} \mathrm{O}_{3}$ can promote dewetting of the liquidus alloy. It is also helpful to choose the substrate such that it is the lowest surface energy system relative to both material X and material Y.

For common liquids with a high contact angle the transition from film to islands/droplets is almost instantaneous. However such a transition in ultrathin liquid films at high temperature is an activated process that involves fluctuation in the film to drive the morphological instability. A thin liquid film represents a morphologic metastable state ${ }^{21}$. It has been shown that ultrathin films of silicon $<50 \mathrm{~nm}$ are morphologically unstable when heated on silicon dioxide substrates with an estimated liquid contact angle of $\sim 73^{\circ} 22,23$. In order to undergo the morphological transition from a wetting film to a dewetting agglomeration state a substrate-exposing perturbation is required.

This perturbation refers to the formation of a void via a fluctuation in the thickness of the thin film exposing a region of the low energy substrate. When the size of this region reaches a certain critical length $\left(r_{\text {crit }}\right)$ there is a decrease in free energy as the void grows $\left(r_{\text {void }}>r_{\text {crit }}\right)$. 
The activation energy required to nucleate such a void is estimated using the following equation (2) 23 .

$$
\Delta G_{\text {void }}=2 \pi r_{\text {crit }} t_{f i l m} \gamma_{f i l m}+\pi r_{\text {crit }}^{2}\left(\gamma_{\text {sub }}-\gamma_{\text {film }}\right)
$$

Where $\Delta G_{v o i d}$ is the activation energy for creating a void large enough to cause dewetting, $r_{c r i t}$ is the size of the critical void $\left(r_{c r i t}=\frac{t_{S i}}{\sin \theta_{S i}}\right), t_{f i l m}$ is the thickness of the film, $\gamma_{f i l m}$ is the surface energy of the film and $\gamma_{\text {sub }}$ is the surface energy of the exposed substrate. For small fluctuations in film thickness the first term in equation (2) dominates. For Silicon on an Insulator (SOI) substrate, taking $r_{c r i t}=t_{f i l m}=1 \mathrm{~nm}, \gamma_{S i}=1.5 \mathrm{~J} / \mathrm{m}^{2}$ the activation energy required to nucleate a critical void $r_{\text {crit }}^{E A} \sim 60 \mathrm{eV}$, such that homogeneous spontaneous nucleation of critical voids does not occur. Void nucleation therefore must be nucleated at either surface/film defects or film edges. Film edges represent pre-existing critical voids of infinite void thickness 23. Film edge effects, for example, can be exploited during bilayer film deposition to promote eutectic liquidus film dewetting and can be achieved experimentally by half covering the substrate $\mathrm{X}$ with $\mathrm{Y}$ bilayer material during deposition so as to dramatically promote dewetting at the film edge as seen in Figure 4. Intentionally milling ion-beam defects into the XY bilayer film prior to liquidus alloy formation also promotes film rupture at that point. The second term in equation (2) becomes more relevant as thickness fluctuations increase in magnitude and the exposed surface area of the surface leads to a free energy reduction since $\gamma_{\text {sub }}<\gamma_{\text {film. }}$.

The artificial introduction of defects can be coupled with another thin film rupture mechanism driven by an attraction (predominantly Van der Waals) interaction between the liquid film and the substrate to exercise greater control over the dewetting and agglomeration of the thin film. This spontaneous film rupture process is most strongly influenced by variations in the thickness of the liquid on the substrate. Liquid surface perturbations occur spontaneously 
in the film and arise from thermal energy and vibration. Their interaction with the film introduces surface interfacial waves that have a characteristic wavelength $\lambda$. Any interfacial wave in the liquid causes a local change in thickness in the film, illustrated in Figure 3 (b), increasing the high energy film-vacuum surface area and so increase that component of the free energy that will drive the opening of voids at the lower energy substrate. The interfacial interaction is captured by the disjoining pressure $\Pi$ (3) which can be thought of as a pressure applied at the boundaries of the film ${ }^{24}$. The disjoining pressure is a function of the film thickness $t$ (height) and describes the intermolecular forces of interaction between a thin film and the substrate.

$$
\Pi=A / 6 \pi t^{3}
$$

Where $A$ is the Hamaker constant. The disjoining pressure is used to derive the critical wavelength $\lambda_{\text {crit }}$ for thin liquid film breakup given below (4).

$$
\lambda_{\text {crit }}=\left(-\frac{2 \pi^{2} \gamma_{f i l m}}{\mathrm{~d} \Pi / \mathrm{d} t}\right)^{1 / 2}
$$

The critical wavelength $\lambda_{\text {crit }}$ is the shortest wavelength of an interfacial perturbation that satisfies the film rupture condition $\mathrm{d} \Pi / \mathrm{d} t>0$ for a set film thickness. Its most general form is given in equation (5).

$$
\lambda_{c r i t}=t^{2} \sqrt{\frac{c \gamma_{f i l m}}{A}}
$$


(a)
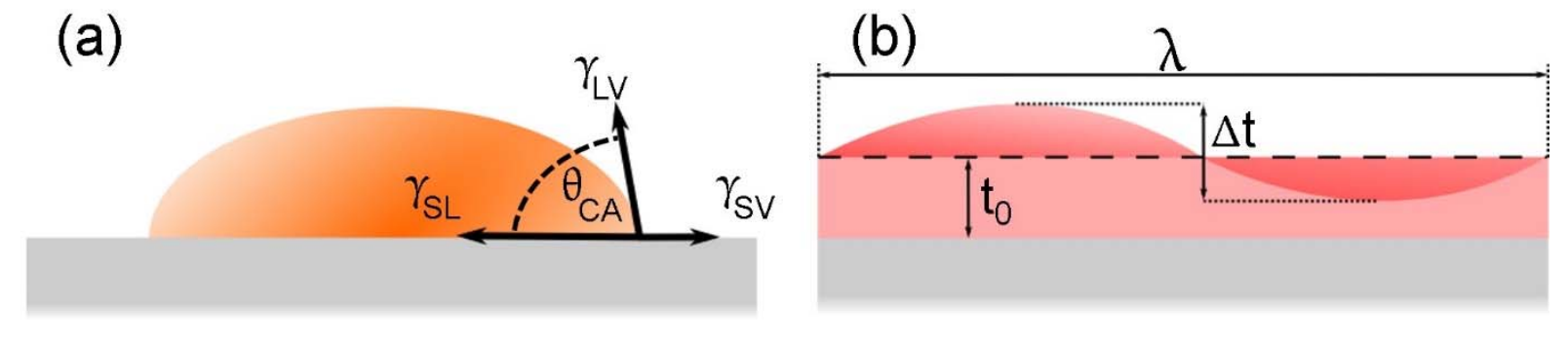

Figure 3 (a) The various surface energy interfaces and equilibrium contact angle considered in Youngs equation are shown. With $\gamma_{\mathrm{SV}}, \gamma_{\mathrm{SL}}$ and $\gamma_{\mathrm{LV}}$ are the surface energies for the solidvapour, solid-liquid and liquid-vapour interfaces and $\theta_{\mathrm{CA}}$ is the equilibrium contact angle. (b) An illustration of a interfacial wave in a thin liquid film oscillating at a wavelength $\lambda$ showing the change in film thickness $\Delta \mathrm{t}$ resulting from a perturbation, where $\mathrm{t}_{0}$ is the initial film thickness. For any set thickness of an ultrathin film there is a range of wavelengths whose amplitudes will continue to grow resulting until $\Delta \mathrm{t}>\mathrm{t}_{0}$ causing film rupture.

Where $c$ is a constant that contains pi $\left(4 \pi^{3}\right)$ as derived by Vrij ${ }^{25,26}$. Perturbation wavelengths $\lambda \geq \lambda_{\text {crit }}$ are amplified resulting in spontaneous rupture of the film ${ }^{27}$. The critical wavelength tends to infinity when $\mathrm{d} \Pi / \mathrm{d} t=0$, this condition is satisfied at the critical thickness $t_{\text {crit }}$ where the film is stable against rupture by this mechanism. Equation (5) can be recast in a more simplified form.

$$
t_{c r i t}=\left(\frac{A \lambda_{c r i t}^{2}}{c \gamma_{f i l m}}\right)^{1 / 4}
$$

Thus any liquid film thinner than the critical thickness described in (6), in the presence of an attractive interaction between itself and the substrate has a set of perturbation wavelengths which will be amplified leading to film rupture. The fastest growing perturbation wavelength is one slightly longer than the critical wavelength. While all wavelengths longer than the critical 
wavelength can cause film rupture not all wavelengths are likely to form ${ }^{28}$. Longer wavelengths have a greater driving force but require more of the liquid to be displaced encountering more viscous friction while shorter wavelengths encounter less viscous friction but have less driving force resulting in low liquid flow speed.

These interfacial waves are the physical origin of the agglomeration waves Jung et al first observed during processing ${ }^{14}$. Figure 4 (a), (b) captures how ion-beam lithography can be used to dictate growth. The critical wavelength for film rupture can be calculated for a set thickness of thin film. Fingerprints of these wavelengths are observed post annealing as shown in Figure 4 (c) for a $20 \mathrm{~nm}$ thick film composed of $10 \mathrm{~nm} \mathrm{Au} / 10 \mathrm{~nm}$ Ge. Using the I-beam milled points as the center, the distance from the center to the agglomerated crystal structures forms a circle of diameter $10 \mathrm{um}$. This value is close to the calculated critical wavelength (equation (6)) of $15 \mu \mathrm{m}$ for a $20 \mathrm{~nm}$ thick AuGe film (10/10 nm Au/Ge, surface energy of liquidus AuGe alloy with 24.5-29.5 atom $\% \mathrm{Ge}$ at $\left.400^{\circ} \mathrm{C}-\gamma_{\mathrm{AuGe}}=1.1 \mathrm{~J} / \mathrm{m}^{2},{ }^{29} \mathrm{~A}=10^{-20} \mathrm{~J},{ }^{30} c=124\right)$. Interpreting this range of wavelengths in the context of controlling the dewetting process is a challenge, however it may be used to construct an ion beam milling pattern in the film prior to annealing that can drive dewetting and agglomeration to optimise wire positioning and growth. Figure 4 (d), (e) captures how the calculated critical wavelength for a given film thickness may be used in cooperation ion-beam milled features to enhance dewetting control.

The critical wavelength is useful for designing a milling pattern to promote positional growth, as seen in Figure 4 (a). It may be used to estimate the distance the liquidus alloy will dewet from the milling site. It can therefore be used to dictate dewetting to position the growth droplets for subsequent nanowire growth. Milling into the deposited films such that the substrate is not exposed (as shown in Figure $4(\mathrm{~d})$ ) introduces defects into the film which act as crystal nucleation points for growth. However, partially milling through some of the bilayer requires careful optimization of the ion beam dose parameter optimization. On the other hand 
by milling to a depth to expose the underlying substrate the though hole acts as a dewetting site as seen in Figure 4 (e). Evidence in literature suggests strongly that lithography and thin film patterning may also be applied with SEDG to dictate dewetting and promote position control $31-34$.

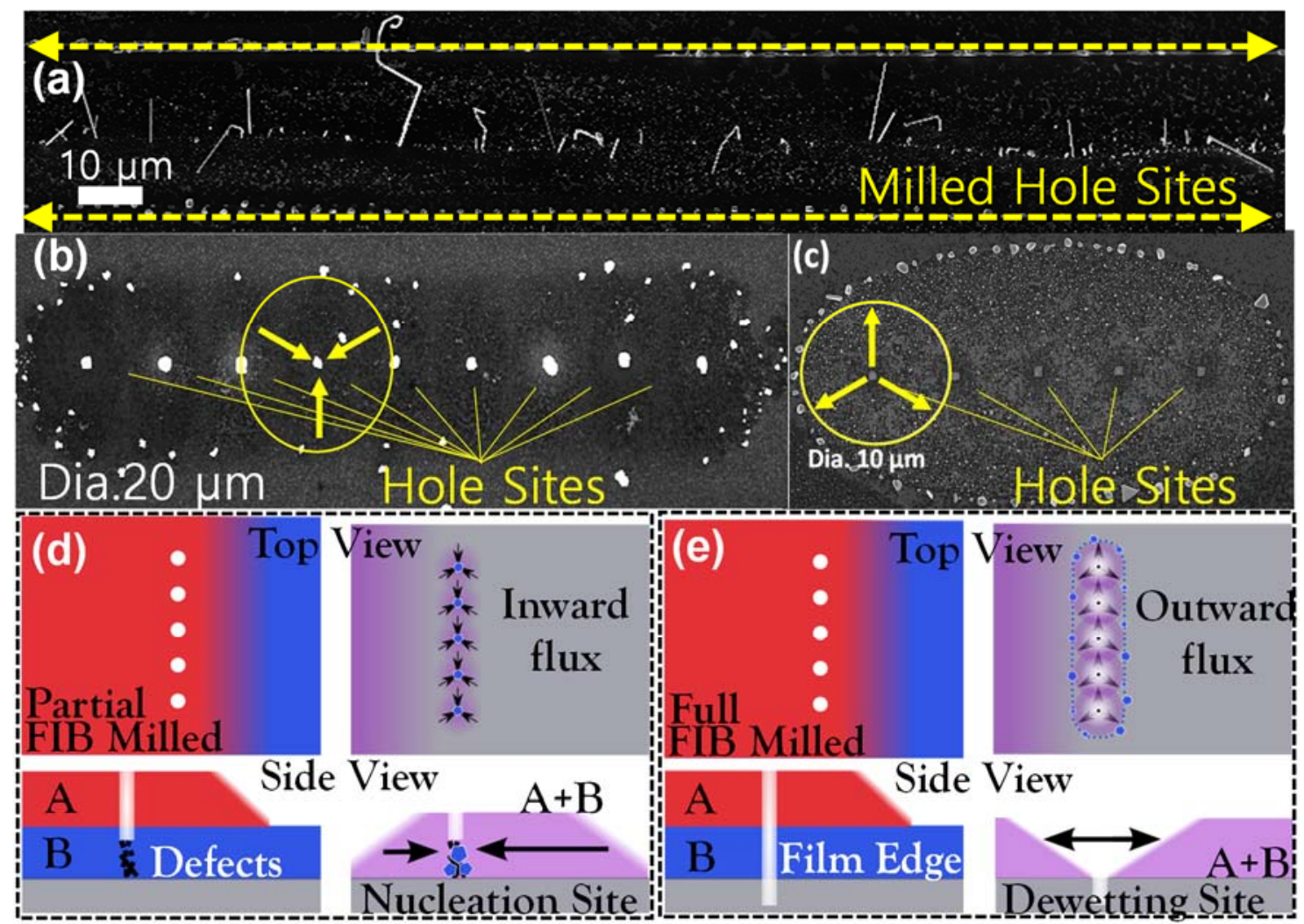

Figure 4 Interfacial waves as the origin of the agglomeration waves first observed by Jung et al ${ }^{14}$ demonstrate the ability to control the direction of dewetting to promote growth. (a) Two rows of I-beam milled hole sites shown in yellow are used to direct dewetting in the bilayer film during annealing. (b) An array of partially I-beam milled holes act as crystal nucleation points for wire growth. The partially milled hole sites produce an inward flux seen in schematic (d). (c) Full I-beam milled holes serve as dewetting sites with an outward flux of material seen in schematic (e). The ability of the holes to 'push' material about the substrate is given by $\lambda_{\text {crit }}$ and can be roughly calculated using equation (6) and the bilayer thickness, in this case for a 10 $\mathrm{nm} \mathrm{Au} / 10 \mathrm{~nm}$ Ge bilayer the $\lambda_{\text {crit }}$ is $15 \mu \mathrm{m}$. (d),(e) Schematics illustrating the difference 
between partially and full milled I-beam holes and the effect they have on dewetting during annealing.

\section{Agglomeration \& Crystal Nucleation}

The role of surface energy in determining thin film stability on a substrate as well as the dynamics of thin film break up have been discussed. These steps facilitate the formation of islands (droplets) on the chosen substrate. The islands share many similarities to the metal catalysts in VLS growth as they represent a low energy surface for adatoms and molecules to adsorb onto relative to the bare substrate and facilitate the decomposition or dissolution of these materials into the droplet. Nucleation and growth of the crystal phase nanostructures proceeds in these droplet islands which is driven by a reduction in free energy of the system.

Classical nucleation theory and the growth of a new crystal phase proceeds via the stepwise accretion of atoms to a critical nucleus ${ }^{35,36}$. The simultaneous accretion of sufficient atoms to the critical nucleus to make it stable against dissolution is highly improbable ${ }^{35,37}$. The change in free energy of the crystal in the growth medium is given below (7).

$$
\Delta G=\Delta G_{S}+\Delta G_{V}
$$

$\Delta G_{S}$ and $\Delta G_{v}$ are the energy needed to create the surface area of the crystal surface and the energy required to create the crystal volume. An expression (8) for the change in free energy as a function of crystal nucleus radius $r$ is given by, 


$$
\Delta G=4 \pi r^{2} \Delta G_{S}+\frac{4}{3} \pi r^{3} \Delta G_{v}
$$

Both energy terms are a function of radius $r$ with the interfacial energy $\gamma_{\text {interface }}=\mathrm{dG} / \mathrm{dA}$ between the new crystal phase and the growth medium influencing the first term. A plot of each term in (8) Figure 5 reveals how the two contribute to the overall free energy change as the size of the nucleus increases, mainly that $\Delta G_{S}$ is always positive and $\Delta G_{V}$ is always negative. There is a critical size $\left(r_{C r i t}\right)$ the nucleus must reach before the $\Delta G_{V}$ term overcomes the $\Delta G_{S}$ term which is dominant for nuclei smaller than $r$ crit. Nucleus equal to or greater in size than $r_{C r i t}$ are deemed critical nucleus, any nucleus larger or equal to this size has an associated decrease in free energy as it grows.

When applied to SEDG, classical nucleation takes place inside the droplets that form after annealing the thin film to the liquidus eutectic temperature. The critical nucleus forms in the liquidus alloy droplet (shown inset Figure 5 (b)). Crystal growth in the liquidus alloy deviates from classical theory due to the differences in surface energy associated with growth of a new crystal phase in vacuum and the liquidus alloy. This surface energy difference will affect the critical nucleus size allowing it to grow larger than that predicted standard classical crystal growth in vacuum as indicated in Figure 5 (a) by the two $\Delta G_{S}$ lines.

Upon the initial breakup of the binary liquidus alloy its composition is assumed to be that of the eutectic. The size of the islands dynamically changes as accretion, dissolution and Ostwald ripening takes place. At this point the relative diffusion rates of the two materials $\mathrm{X}$ and $\mathrm{Y}$ becomes important. The conditions for saturation, nucleation and growth of X-type material are met when the relative rate of diffusion of X-type adatoms to the liquid droplet is greater than that of the Y-type adatoms. Furthermore the high surface energy component segregates 
from the low surface energy component via Gibbs-Thomson pressure associated with growth from a high curvature surface (the liquidus island). With the saturation condition met, the composition of the liquidus alloy begins to change. This composition change can be followed on the XY alloy phase diagram in Figure 5. As the droplet becomes more saturated a critical nucleus forms within it. With continued heating growth of the nucleus can proceed and the single crystal nanostructure forms. One advantage of SEDG is the ability to capture this crystal growth in real time as there is no required forming or precursor gas flow needed to grow the single crystal. Therefore it is possible to capture annealing in real time within the SEM chamber and gain novel insight into the nucleation dynamics and kinetics as seen in the next section.

In the final stages of crystal growth the size of the nanostructures is determined by the amount of material deposited in the thin film. The upper limit of the binary film thickness is set by its stability. A very thick film will simply be too stable to rupture and undergo agglomeration. A unique feature of SEDG is the presence of a capping layer which surrounds the single crystal nanostructure core which is similar in many ways to the metal catalysts that remain on top of nanowires grown via VLS methods. The SEDG capping layers are formed by the condensation of the liquidus eutectic droplet during annealing. 
(a)

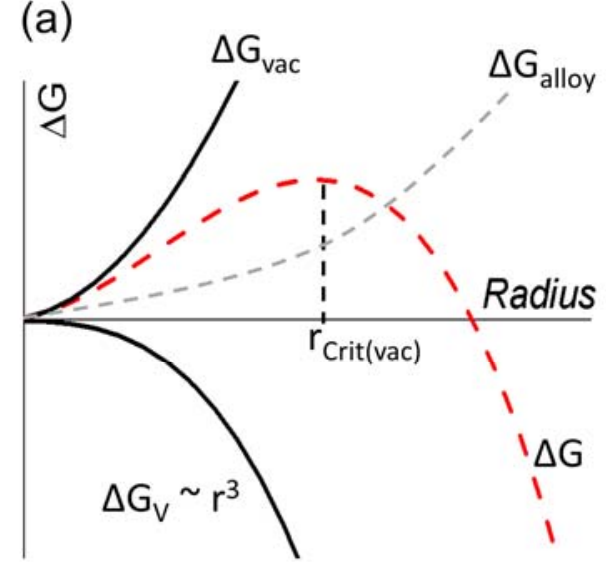

(b)

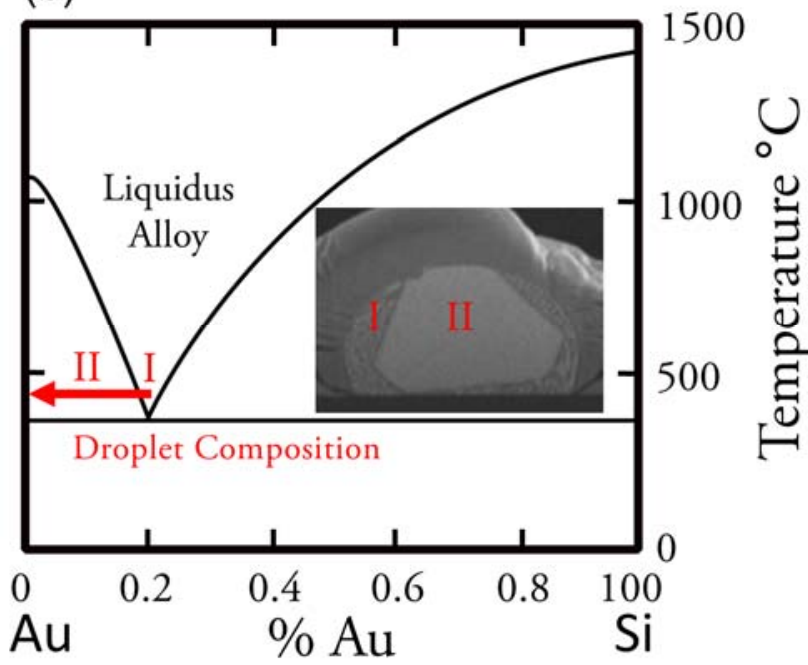

Figure 5 (a) Free energy change of a spherical nucleus as a function of its radius showing the contributions of each term in equation (8), $\Delta \mathrm{Gs}$ and $\Delta \mathrm{Gv}$ (solid lines), with a plot of the two terms together (dashed red line) highlighting the presence of a nucleation barrier for nuclei with a subcritical size $r<$ rcrit. The size of the stable nucleus is a function of surface energy at the interface between the new crystal phase and the growth medium. During SEDG nucleation liduidus alloy droplets of eutectic composition will surround the new crystal increasing the $\mathrm{r}_{\text {Crit }}$ size, indicated by the dashed grey line. (b) Binary alloy phase diagram of Au and Si depicting the change in droplet composition as Au adatoms continue to saturate an AuSi liquidus droplet forming during annealing. At point I the composition is that of the eutectic, at point II the composition of the droplet is liquidus eutectic $+\mathrm{Au}$ crystal phase. Inset shows the new crystal phase $(\mathrm{Au})$ encased by the liquidus alloy (AuGe). 


\section{Real Time Nucleation \& Metastable Crystal Nucleus}

There are several assumptions about the nature of critical nucleus in classical nucleation theory ${ }^{38}$. Most notably the shape of the particles formed are assumed to be spherical and that these nuclei are ordered in the same arrangement as the crystal phase that will eventually form. The growth process is also assumed to proceed in a stepwise manor which doesn't account for the possible combination of subcritical nuclei. Classical nucleation theory also fails to explain nucleation phenomena such as poor nucleation rates of crystals in undercooled liquids and gas phase nucleation in superheated liquids ${ }^{39}$. A two-step nucleation process was proposed in which the first step is the formation of aggregate clusters in solution followed by the nucleation of ordered crystalline solids within these clusters ${ }^{40}$. The formation of clusters or metastable nuclei is believed ${ }^{41}$ to lower the free-energy barrier for the growth of the stable critical nucleus. A schematic representation of the two-step nucleation process is shown in Figure 6 (a). It describes a proposed alternate pathway for the formation of a critical sized nucleus via the combination of atoms in crystal shapes not representative of the final most stable nucleus. The two-step mechanisms suggests a nucleus approaching $r_{\text {Crit }}$ in size, it will transition into the final stable form of the growing nanostructure.

Current methods used to investigate small cluster formation during nucleation include dynamic light scattering (DL S), UV-visible and X-ray spectroscopy ${ }^{42}$. The direct observation of metastable states remains difficult owing to the transient nature and lifetime of metastable nuclei. TEM has been used to capture and monitor crystal growth. ${ }^{43,44}$ By heating the bilayer $\mathrm{XY}$ film on an SEM hotstage it is possible to monitor the growth of the nanocryrstals in real time with SEM. As seen in Figure 6 (b) for the real time growth of Fe crystals via SEDG - the 
shape of the growing crystal changes rapidly from pyramidal to cubic through the course of the experiment. As evidenced by the crystal shape change during growth the relatively large crystal supports a larger than normal critical nucleus size as discussed in the previous section. This method of hot stage SEM or TEM annealing coupled with SEDG growth could potentially be used to elucidate/observe two step nucleation or pre-nucleation processes.

(a)
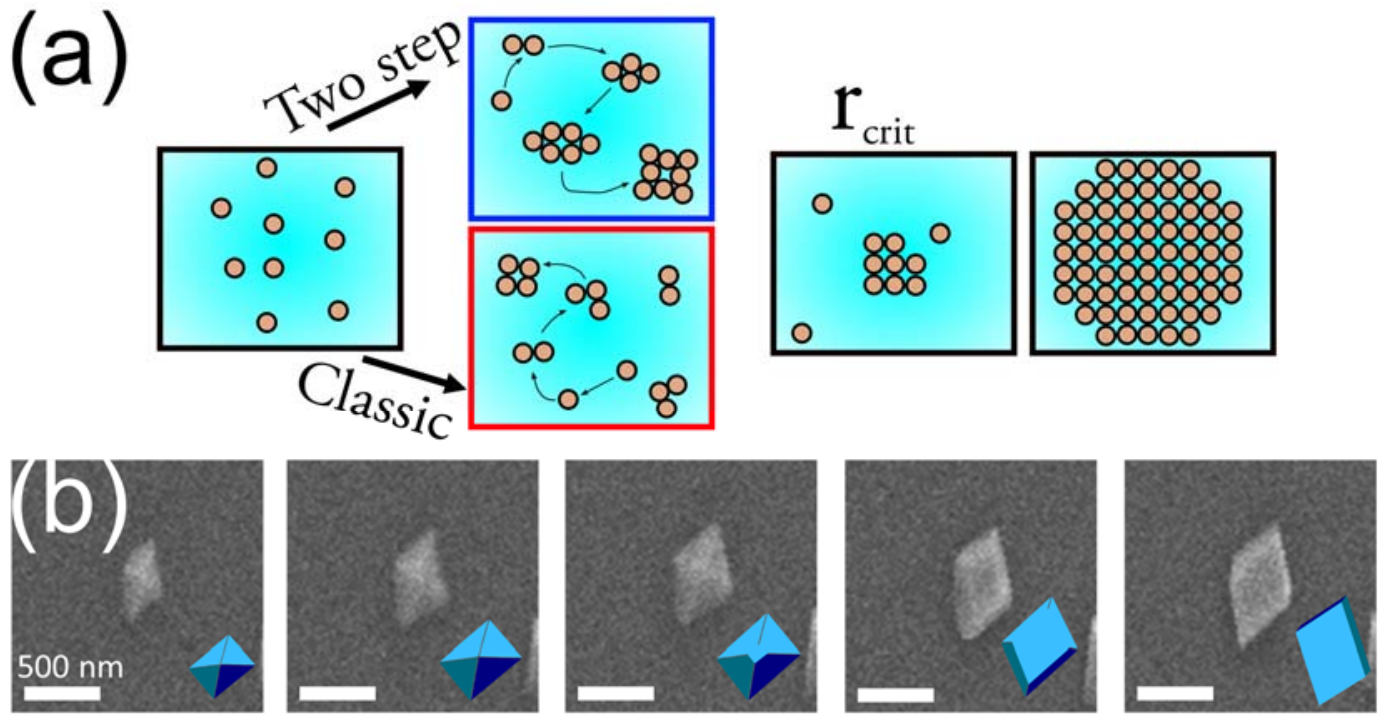

time

0:00 m:ss

$0: 38$

$1: 05$

$1: 28$

2:05 
Figure 6 (a) A schematic describing each step in classical and the so called two step nucleation process. Classical nucleation is introduced as the piecewise addition of individual atoms to a growing nucleus whose shape is the same as the final stable nucleus. The two step nucleation process describes the piecewise addition of atoms to a growing nucleus that is metastable and transient. The role of the meta-stable nucleus is to facilitate growth up to a critical nucleus size where the metastable nucleus then transforms into its final most stable crystal shape for continued growth. (b) The growth of nanostructures can be monitored in real time with SEDG using an SEM with a hotstage. The sequence of SEM images follows the evolution of an Fe crystal from its initial pyramidal crystal shape to that of a cubic crystal. The final shape of the crystal is determined by the lowest energy crystal surface $-<100>$ cubic for Fe crystals. It is calculated using Wulff construction and is an essential tool in predicting the final form the nanostructure will take when using SEDG with other materials. Monitoring the growth in real time reveals the transition between different crystal nuclei shapes. The accessibility of SEDG and hot stage SEM monitoring could prove useful in elucidating these crystal nuclei shape transitions during growth.

\section{Summary and Outlook of SEDG}

The SEDG method is a novel and inexpensive bottom up synthesis method for producing very large single crystal metal nanostructures that can be prepositioned on a substrate. The method involves depositing two thin films of a binary alloy system and heating them above their eutectic temperature to form a liquidus alloy. The surface energies of the system are chosen to promote the liquidus eutectic breakup to allow saturation then nucleation to occur. The potential of SEDG may be unlocked when used in conjunction with top down fabrication 
techniques such as pulsed laser deposition, nanoindentation and e-beam lithography or I-beam lithography to more accurately position growth of the nanostructures. I-beam lithography has been shown to control dewetting of the liquidus film by either partially milling into the film prior to annealing creating defect sites or by milling through the film to act as pinning sites for the so call agglomeration wavefronts. The length of wavefronts can be calculated and therefore allow a milling pattern to be defined such that the dewetting of the film can be controlled for promoting nanostructure growth. When heated on a hotstage in an SEM chamber the SEDG method offers a glimpse in real time at the growth kinetics of the nanostructures which may reveal novel nucleation phenomena such as metastable nucleation states.

To date the SEDG method has been applied to over 3 separate systems to produce metal nanostructure growth. It's possible that the method can be applied to a wealth of binary alloy systems to produce unique nanostructures that cannot be realised easily through other growth methods. The binary alloy system allows significant reduced processing temperatures relative to the individual melting temperatures of the system. Adding a third alloy material to form a ternary alloy system could also be used to further depress processing temperatures. The main areas the SEDG method could be applied to include inexpensive catalysis formation, hierarchical advance materials and the production of very large single crystal metal nanostructures relative to other synthesis methods.

\section{Acknowledgements}

We acknowledge the Science Foundation Ireland funded Principal Investigator Award (Grant No. 06/IN.1/I106) and support from the European Research Council under Advanced Grant 321160. 


\section{References}

(1) Hobbs, R. G.; Petkov, N.; Holmes, J. D. Chemistry of Materials 2012, 24, 1975.

(2) Wagner, R.; Ellis, W. Vapor-liquid-solid mechanism of crystal growth and its application to silicon.

(3) Morales, A. M.; Lieber, C. M. Science 1998, 279, 208.

(4) Yu, D. P.; Bai, Z. G.; Ding, Y.; Hang, Q. L.; Zhang, H. Z.; Wang, J. J.; Zou, Y. H.; Qian, W.; Xiong, G. C.; Zhou, H. T.; Feng, S. Q. Applied Physics Letters 1998, 72,3458 .

(5) Wang, Y.; Meng, G.; Zhang, L.; Liang, C.; Zhang, J. Chemistry of Materials 2002, 14, 1773.

(6) López-López, M.; Guillén-Cervantes, A.; Rivera-Alvarez, Z.; HernándezCalderón, I. Journal of Crystal Growth 1998, 193, 528.

(7) Shimada, T.; Hiruma, K.; Shirai, M.; Yazawa, M.; Haraguchi, K.; Sato, T.; Matsui, M.; Katsuyama, T. Superlattices and Microstructures 1998, 24, 453.

(8) Hiruma, K.; Yazawa, M.; Katsuyama, T.; Ogawa, K.; Haraguchi, K.; Koguchi, M.; Kakibayashi, H. Journal of Applied Physics 1995, 77, 447.

(9) Wu, X. C.; Song, W. H.; Zhao, B.; Sun, Y. P.; Du, J. J. Chemical Physics Letters 2001, 349, 210.

(10) Liu, X.; Li, C.; Han, S.; Han, J.; Zhou, C. Applied Physics Letters 2003, 82, 1950.

(11) Young Kim, H.; Park, J.; Yang, H. Chemical Physics Letters 2003, 372, 269.

(12) Ma, R.; Bando, Y. Chemistry of Materials 2002, 14, 4403.

(13) Young Kim, H.; Park, J.; Yang, H. Chemical Communications 2003, 256.

(14) Jung, S. J.; Lutz, T.; Boese, M.; Holmes, J. D.; Boland, J. J. Nano Lett 2011, 11, 1294.

(15) Curtis, O. K.; Soon Jung, J.; Alan, P. B.; John, J. B. Nanotechnology 2012, 23, 435604.

(16) Jung, S. J.; Lutz, T.; Bell, A. P.; McCarthy, E. K.; Boland, J. J. Crystal Growth \& Design 2012, 12, 3076.

(17) O’Regan, C.; Biswas, S.; O'Kelly, C.; Jung, S. J.; Boland, J. J.; Petkov, N.; Holmes, J. D. Chemistry of Materials 2013, 25, 3096.

(18) Mills, K. C.; Su, Y. C. International Materials Reviews 2006, 51, 329.

(19) Okamoto, H. ASM International 2010, 44.

(20) Mills, K.; Su, Y. International Materials Reviews 2006, 51, 329.

(21) WW, M. Journal of Applied Physics 1959, 30, 77.

(22) R. Nuryadi, Y. I., M. Tabe Applied Surface Science 2000, 160.

(23) Danielson, D. T.; Sparacin, D. K.; Michel, J.; Kimerling, L. C. Journal of Applied Physics 2006, 100, 083507.

(24) Dai, B.; Leal, L.; Redondo, A. Physical Review E 2008, 78.

(25) Vrij, A. Discuss. Faraday Soc. 1966, 42, 23.

(26) Sheludko, A. Adv Colloid Interface Sci 1967, 1, 391.

(27) Saramago, B. Current Opinion in Colloid \& Interface Science 2010, 15, 330.

(28) Manev, E. D.; Nguyen, A. V. Adv Colloid Interface Sci 2005, 114-115, 133.

(29) Sa, I.; Lee, B.-M.; Kim, C.-J.; Jo, M.-H.; Lee, B.-J. Calphad 2008, 32, 669.

(30) F. Vandenbrouck, M. P. V., A.M. Cazabat Physical Review Letters 1999, 82.

(31) Claudia Manuela, M.; Flavio Carlo Filippo, M.; Ralph, S. Nanotechnology 2008, 19, 485306. 
(32) Tan, B. J. Y.; Sow, C. H.; Koh, T. S.; Chin, K. C.; Wee, A. T. S.; Ong, C. K. The Journal of Physical Chemistry B 2005, 109, 11100.

(33) Lin, J.; He, W.; Vilayurganapathy, S.; Peppernick, S. J.; Wang, B.; Palepu, S.; Remec, M.; Hess, W. P.; Hmelo, A. B.; Pantelides, S. T.; Dickerson, J. H. ACS Applied Materials \& Interfaces 2013, 5, 11590.

(34) Bechelany, M.; Maeder, X.; Riesterer, J.; Hankache, J.; Lerose, D.; Christiansen, S.; Michler, J.; Philippe, L. Crystal Growth \& Design 2010, 10, 587.

(35) Sangwal, K. Additives and Crystallization Processes from Fundamentals to Application; Wiley, 2007.

(36) Sunagawa, I. Crystals: Growth, Morphology and Perfection, 2007.

(37) Sun, Y. Chemical Society reviews 2013, 42, 2497.

(38) Deniz Erdemir, A. Y. L., Allan S. Myerson Accounts of Chemical Research 2009, $42,621$.

(39) Oxtoby, D. W. Accounts of Chemical Research 1998, 31, 91.

(40) Filobelo, L. F.; Galkin, O.; Vekilov, P. G. J Chem Phys 2005, 123, 014904.

(41) James F. Lutsko, G. N. Physical Review Letters 2006, 96.

(42) Finney, E. E.; Finke, R. G. Journal of colloid and interface science 2008, 317, 351.

(43) Liao, H.-G.; Cui, L.; Whitelam, S.; Zheng, H. Science 2012, 336, 1011.

(44) Wu, Y.; Yang, P. Journal of the American Chemical Society 2001, 123, 3165. 